\title{
Influência da hidrocortisona no desenvolvimento do Schistosoma mansoni em Biomphalaria glabrata
}

\author{
Influence of hydrocortisone on Schistosoma mansoni \\ development in Biomphalaria glabrata \\ Deborah Regina Serrano, Eliana Maria Zanotti-Magalhães, \\ Luiz Augusto Magalhães e José Ferreira de Carvalho
}

\begin{abstract}
Resumo Foi avaliado o efeito da hidrocortisona no desenvolvimento do Schistosoma mansoni da linhagem $\mathrm{BH}$ em Biomphalaria glabrata. Moluscos escolhidos ao acaso foram tratados com hidrocortisona durante quatro dias. No segundo dia de tratamento, os moluscos foram expostos a 10 miracídios de S. mansoni. Um outro grupo, não tratado com hidrocortisona, foi exposto aos miracídios e observados da mesma forma. Os seguintes dados foram observados: taxa de infecção, tempo de sobrevivência, período pré-patente e número de cercárias liberadas. Os resultados mostraram que os moluscos no grupo tratado com hidrocortisona apresentaram maior taxa de infecção, menor período pré-patente, maior produção de cercárias e maior tempo de sobrevivência. Estas observaç̃es sugerem que a hidrocortisona atuou nos moluscos diminuindo a resistência à infecção esquistossomótica, facilitando o desenvolvimento do trematódeo, atenuando os efeitos lesivos da infecção e proporcionando maior sobrevivência.
\end{abstract}

Palavras-chaves: Schistosoma mansoni. Biomphalaria glabrata. Hidrocortisona. Esquistossomose.

Abstract The effect of hydrocortisone on the development of BH strain of S. mansoni in B. glabrata snails is evaluated. Snails in a randomly chosen group were submitted to hydrocortisone during four days. In the second day of hydrocortisone exposure, the snails were exposed, each, to ten S. mansoni miracidia. Another group, not treated with hydrocortisone, was exposed to miracidia and observed in the same manner. Various items of data were observed: the infection rate, survival time, prepatent period and total number of cercariae released. It was found that the snails in the group treated with hydrocortisone had a higher infection rate, shorter prepatent periods, produced more cercariae and also had longer survival times. This suggests that the hydrocortisone in snails induces a decrease in resistance to schistosome infection and facilitates the development of the trematode, while also reducing adverse infection effects, thereby leading to longer snail survival.

Key-words: Schistosoma mansoni. Biomphalaria glabrata. Hydrocortisone. Schistosomiasis.

No presente trabalho estudamos alguns aspectos da ação da hidrocortisona sobre o desenvolvimento do Schistosoma mansoni da linhagem BH em B. glabrata. Os glicocorticóides, dentre eles a hidrocortisona, exercem nos mamíferos, efeitos sobre o metabolismo das proteínas, gorduras e dos hidratos de carbono, sobre o balanço hídrico e de eletrólitos. Entre os efeitos, observa-se o aumento da síntese de glicogênio, com acentuado acúmulo deste carboidrato no fígado. Sobre as proteínas, promove a sua quebra e inibe seu anabolismo e síntese, interferindo na cicatrização de feridas e inibindo a produção de anticorpos ${ }^{3}{ }^{4}$. A ação anti-inflamatória e imunossupressora nos mamíferos torna a hidrocortisona freqüentemente utilizada desta forma, em medicina, quando se deseja atenuar os efeitos indesejáveis dos processos inflamatórios e alérgicos ${ }^{9}$. Os efeitos da hidrocortisona observados nos mecanismos de defesa dos mamíferos, principalmente sobre as células fagocitárias e no metabolismo de hidratos de carbono nos induziram a verificar a possível influência da hidrocortisona no desenvolvimento da infecção do $S$. mansoni em moluscos vetores tratados por esse glicocorticóide.

\footnotetext{
Departamento de Parasitologia do Instituto de Biologia da Universidade Estadual de Campinas, Campinas, SP; Statistika Consultoria, Campinas, SP. Financiado pela Coordenação de Aperfeiçoamento de Pessoal de Nível Superior (CAPES).

Endereço para correspondência: Dr. Luiz Augusto Magalhães. Dept ${ }^{\circ}$. de Parasitologia/IB UNICAMP, Caixa Postal 6109 , Cidade Universitária, Barão Geraldo, 13083-970 Campinas, SP, Brasil.

Telefax:55 19 3788-6282

e-mail: lam@obelix.unicamp.br

Recebido em 19/5/2000.
} 
Neste estudo pesquisamos a influência da hidrocortisona sobre a taxa de infecção de B. glabrata, a sobrevivência dos moluscos expostos à infecção e sobre a produção de cercárias.

\section{MATERIAL E MÉTODOS}

Foram utilizados exemplares de Biomphalaria glabrata albinos com diâmetro variando de 7 a $8 \mathrm{~mm}$, originados de populações de Belo Horizonte (MG, Brasil), mantidos em nossos laboratórios. A infecção dos moluscos foi realizada com Schistosoma mansoni da linhagem $\mathrm{BH}^{6}$ obtida de camundongos infectados experimentalmente. Foram constituídos quatro grupos experimentais, em uma estrutura fatorial com dois fatores em dois níveis: Grupo I: moluscos não submetidos à infecção e não tratados com hidrocortisona; Grupo II: moluscos não submetidos à infecção e tratados com hidrocortisona; Grupo III: moluscos submetidos à infecção e não tratados com hidrocortisona; Grupo IV: moluscos submetidos à infecção e tratados com hidrocortisona. Usou-se um esquema completamente aleatorizado para alocação dos moluscos aos quatro grupos experimentais.

O tratamento com hidrocortisona, que durou quatro dias, foi realizado colocando-se cada subgrupo de 4 moluscos em um recipiente com $100 \mathrm{ml}$ de água declorada e $0,3 \mathrm{ml}$ de solução de succinato sódico de hidrocortisona (Solu-Cortef a $500 \mathrm{mg} / 4 \mathrm{ml}$ ). A cada 24 horas a água foi trocada, adicionando-se nova dose de hidrocortisona. No quinto dia, os moluscos foram colocados, por grupo, em um recipiente único com água declorada. A exposição ao S. mansoni foi feita no segundo dia de tratamento, expondo-se isoladamente cada molusco, de grupos a serem expostos, a 10 miracídios de S. mansoni provenientes de ovos colhidos nas fezes de camundongos infectados com a linhagem BH do trematódeo.
Os 20 exemplares de moluscos que formavam cada um dos quatro grupos iniciais foram examinados durante 23 semanas, sendo anotado o índice de sobrevivência.

Posteriormente ao início do experimento, adicionamos mais 2 grupos de 20 moluscos (um grupo constituído de moluscos submetidos à infecção e não tratados com hidrocortisona: IIla, e outro grupo submetido à infecção e tratado com hidrocortisona: IVa). Esses dois grupos foram adicionados ao experimento com a finalidade de apenas podermos nos certificar da baixa taxa de infecção obtida nos grupos similares anteriormente constituídos. Após quatro semanas da exposição aos miracídios, os caramujos dos grupos III, IV, Illa e IVa foram examinados semanalmente para verificação da liberação das cercárias ${ }^{8}$. Uma vez confirmadas as baixas taxas de infecção em todos os grupos, e devido ao fato do número de cercárias contadas nos grupos III e IV ser suficiente para a análise estatística, não julgamos necessária a contagem das larvas nos grupos IIla e IVa.

As cercárias liberadas pelos moluscos dos grupos III e IV foram contadas, utilizando solução de ninhidrina ${ }^{7}$. Os dados foram analisados estatisticamente, considerando os grupos submetidos à infecção e tratados ou não com hidrocortisona. $\mathrm{Na}$ análise da sobrevivência dos moluscos foi também considerada a exposição ou não à infecção dos moluscos. Os dados foram analisados

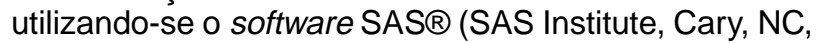
USA). A metodologia empregada está descrita nos resultados para cada resposta analisada.

\section{RESULTADOS}

Na Figura 1 e Tabela 1 estão apresentados os resultados referentes à sobrevivência dos moluscos tratados ou não com hidrocortisona e submetidos ou

Tabela 1 - Medianas de tempos de sobrevivência.

\begin{tabular}{lcc}
\hline Infecção & Tratamento & $\begin{array}{c}\text { Tempo de sobrevivência } \\
\text { mediana (semanas) }\end{array}$ \\
\hline não & não & 13,0 \\
não & sim & 14,0 \\
sim & não & 9,0 \\
sim & sim & 16,5 \\
\hline
\end{tabular}

não à infecção por S. mansoni (Grupos I, II, III e IV). A análise estatística considerando os moluscos expostos ou não à infecção e tratados ou não pela hidrocortisona (em um esquema fatorial $2 \times 2$ ) comparou as medianas das curvas de sobrevivência (estatística Log Rank) e concluiu pela existência de diferença significativa entre estas curvas de sobrevivência $(p=0,03)$. Um teste adicional, retirando o grupo tratado e infectado da comparação, resulta em $\mathrm{p}=0.85$; logo, podemos concluir que há evidência de diferença apenas para o grupo tratado e infectado, com aumento da mediana do tempo de sobrevida. Uma outra comparação, para avaliar o efeito da hidrocortisona apenas entre os infectados, resultou em $p=0.04$ no teste do Log Rank. Note-se que essas seqüências de testes não estão corrigidas para múltiplas comparações, de sorte que os níveis de significância obtidos para as comparações adicionais devem ser vistos apenas como indicativos. Estes resultados todos revelam alguma evidência do efeito da hidrocortisona na sobrevida dos moluscos infectados.

Os dados referentes à taxa de infecção estão apresentados nas Tabelas 2 e 3 . Moluscos não submetidos aos tratamento com hidrocortisona (Grupo III) apresentaram eliminação de cercárias a partir da $6^{a}$. semana após a exposição aos miracídios, observandose eliminação destas larvas até a $13^{\mathrm{a}}$. semana, enquanto moluscos tratados com hidrocortisona (Grupo IV) apresentaram eliminação de cercárias a partir da $5^{\mathrm{a}}$. semana após a infecção, notando-se neste caso, liberação das cercárias até a 19ª semana (Tabela 2). 


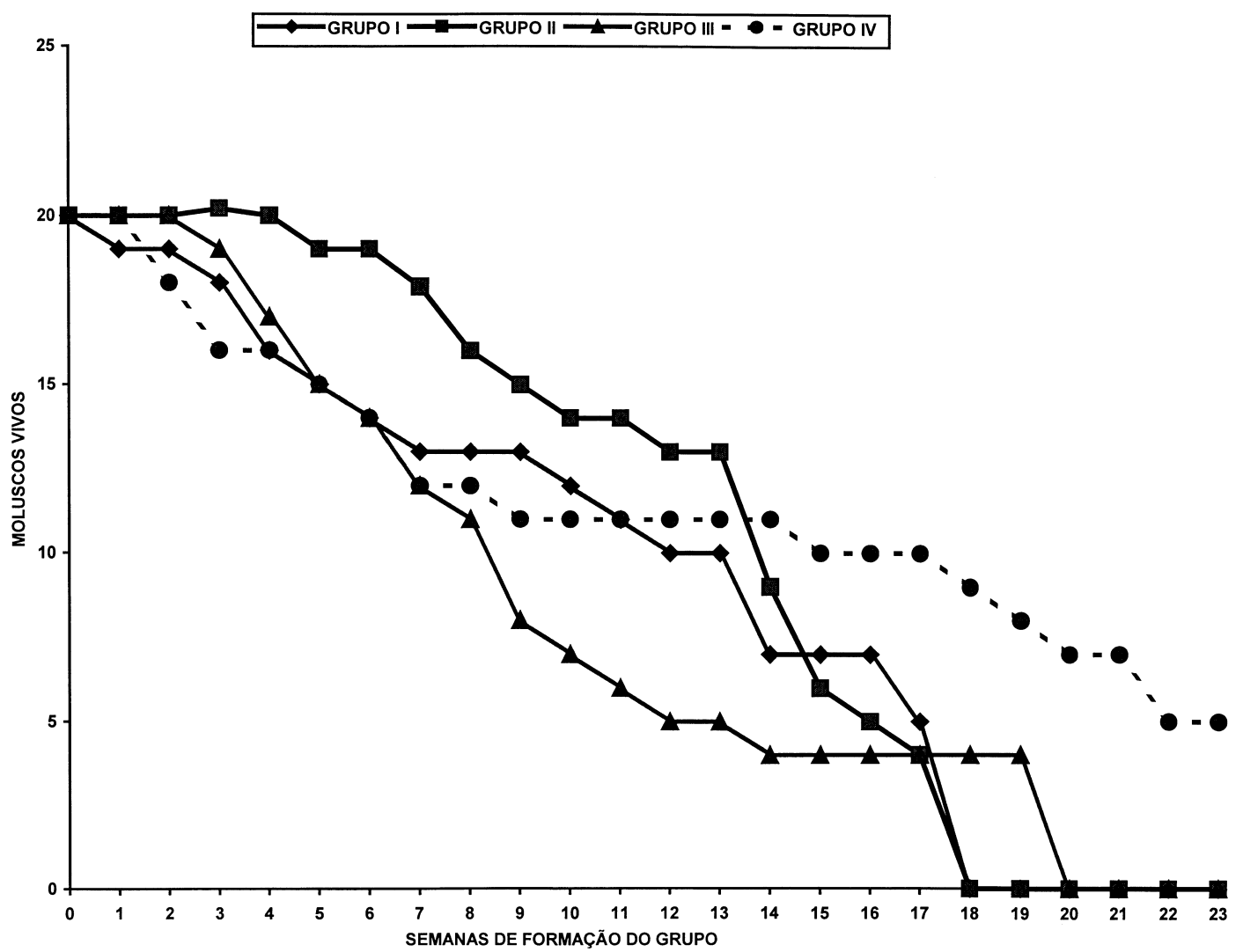

Figura 1 - Curvas de sobrevivências nos grupos I (não submetidos à infeccão, não tratados com hidrocortisona), Il (não submetidos à infecção, tratados com hidrocortisona), Ill (expostos à infecção, não tratados com hidrocortisona) e IV (expostos à infecção, tratados com hidrocortisona) observadas ao longo de 23 semanas de experimento.

\begin{tabular}{|c|c|c|c|c|}
\hline \multirow[b]{2}{*}{ Semanas } & \multicolumn{2}{|c|}{ Grupo III } & \multicolumn{2}{|c|}{ Grupo IV } \\
\hline & moluscos vivos & moluscos positivos & moluscos vivos & moluscos positivos \\
\hline 4 & 35 & 0 & 35 & 0 \\
\hline 5 & 34 & 0 & 33 & 4 \\
\hline 6 & 33 & 2 & 32 & 6 \\
\hline 7 & 31 & 3 & 29 & 3 \\
\hline 8 & 29 & 2 & 27 & 4 \\
\hline 9 & 26 & 1 & 26 & 6 \\
\hline 10 & 25 & 1 & 26 & 5 \\
\hline 11 & 24 & 1 & 26 & 6 \\
\hline 12 & 23 & 1 & 26 & 6 \\
\hline 13 & 21 & 1 & 26 & 6 \\
\hline 14 & 19 & 0 & 23 & 3 \\
\hline 15 & 19 & 0 & 21 & 2 \\
\hline 16 & 18 & 0 & 21 & 2 \\
\hline 17 & 16 & 0 & 21 & 2 \\
\hline 18 & 14 & 0 & 20 & 1 \\
\hline 19 & 13 & 0 & 17 & 1 \\
\hline 20 & 9 & 0 & 14 & 0 \\
\hline
\end{tabular}


Tabela 3 - Taxa de infecção dos moluscos tratados (Grupos IV e IVa) ou não (Grupos III e IIIa) com hidrocortisona e expostos ao S. mansoni da linhagem $\mathrm{BH}$.

\begin{tabular}{lccc}
\hline Grupos & Número de moluscos expostos à infecção & Número de moluscos positivos & Taxa de infecção (\%) \\
\hline III e IIla & 40 & 3 & 7,5 \\
IV e IVa & 40 & 9 & 22,5 \\
\hline
\end{tabular}

Dos moluscos tratados com a hidrocortisona (Grupos IV e IVa), $22,5 \%$ resultaram positivos e $7,5 \%$ dos moluscos não tratados (Grupos III e IIla) se infectaram com o S. mansoni. O teste de Fisher que compara as pequenas proporções (as de respostas positivas) revelou um nível de significância de 0,057 , ou seja, cerca de 6\%. Este valor indica certa significância, mostrando que é razoável admitir que o tratamento com hidrocortisona favoreceu a infecção por S. mansoni.

$\mathrm{Na}$ Tabela 4, estão apresentados os resultados referentes ao número de cercárias eliminadas pelos moluscos tratados (Grupo IV) ou não com hidrocortisona (Grupo III). Os moluscos do grupo IV produziram número significativamente maior de cercárias que os moluscos do grupo III $(p=0,0001)$.

\begin{tabular}{|c|c|c|c|c|c|c|c|c|c|c|}
\hline \multirow[b]{2}{*}{ Semana } & \multicolumn{3}{|c|}{ Moluscos do grupo III } & \multicolumn{6}{|c|}{ Moluscos do grupo IV } & \multirow[b]{2}{*}{ Total } \\
\hline & 1Bglll & 2BgllI & Total & 1BglV & 2BglV & 3BgIV & 4BgIV & 5BgIV & 6BgIV & \\
\hline 5 & - & - & - & 692 & 729 & - & - & - & - & 1.421 \\
\hline 6 & 523 & - & 523 & 1.754 & 1.709 & 692 & - & - & - & 4.155 \\
\hline 7 & 738 & 927 & 1.655 & - & 2.641 & 4.135 & - & - & - & 6.776 \\
\hline 8 & 326 & - & 326 & - & 365 & 4.008 & 711 & - & - & 5.084 \\
\hline 9 & - & - & - & - & - & 2.624 & 326 & - & - & 2.950 \\
\hline 10 & - & - & - & - & - & 2.967 & 1.238 & - & - & 4.205 \\
\hline 11 & - & - & - & - & - & 3.741 & 2.700 & 163 & - & 6.604 \\
\hline 12 & - & - & - & - & - & 3.705 & 2.326 & 398 & - & 6.429 \\
\hline 13 & - & - & - & - & - & 3.727 & 2.077 & 2.596 & - & 8.400 \\
\hline 14 & - & - & - & - & - & 1.129 & 646 & 422 & - & 2.197 \\
\hline 15 & - & - & - & - & - & 4.692 & - & 3.209 & - & 7.901 \\
\hline 16 & - & - & - & - & - & 3.102 & - & 1.715 & - & 4.817 \\
\hline 17 & - & - & - & - & - & 1.902 & - & 2.608 & - & 4.510 \\
\hline 18 & - & - & - & - & - & - & - & 1.456 & - & 1.456 \\
\hline 19 & - & - & - & - & - & - & - & - & 2.614 & 2.614 \\
\hline Total & 1.587 & 927 & 2.514 & 2.446 & 5.444 & 36.424 & 10.024 & 12.567 & 2.614 & 69.519 \\
\hline
\end{tabular}

\section{DISCUSSÃO}

A infecção por S. mansoni produz nos planorbídeos alterações teciduais importantes que podem provocar a morte ${ }^{5}$. Verificamos na Figura 1, um gráfico demonstrativo da mortalidade dos moluscos dos diferentes grupos formados para o experimento, que 0 comportamento referente ao item analisado (sobrevivência) apresentou comportamento diferente após a $13^{\mathrm{a}}$ semana de observação. $\mathrm{Na} 13^{\mathrm{a}}$. semana de observação verificou-se uma maior sobrevivência nos grupos de moluscos não expostos à infecção e tratados, expostos à infecção e tratados e não expostos à infecção e não tratados, sendo que o grupo de moluscos expostos à infecção e não tratados apresentou menor sobrevivência. Essas observações parecem mostrar ter havido uma inibição do processo infeccioso pela ação da hidrocortisona, o que poderia explicar a maior sobrevida dos moluscos submetidos ao tratamento. $\mathrm{Na}$ prática médica a utilização dos glicocorticóides no tratamento das infecções tem que ser feita com cautela, pois a intenção é somente a redução do processo infeccioso em tecidos nobres, reduzindo-se assim. as seqüelas graves. Ainda que doses elevadas dessas substâncias produzam diminuição da ação dos mecanismos de defesa sua administração pode ser justificada em certos processos infecciosos graves, como por exemplo, na tuberculose miliar, na meningite tuberculose e em outros processos ${ }^{10}$. Após a $13^{\mathrm{a}}$ semana a mortalidade do grupo exposto à infecção e não tratado estabilizou-se, talvez em decorrência da diminuição do processo infeccioso ou a própria cura dos moluscos, o que ocorre freqüentemente durante esse período ${ }^{1}$. Não encontramos uma explicação para a alta mortalidade observada a partir da $13^{\mathrm{a}}$. semana no grupo de moluscos não submetidos à infecção e tratados com hidrocortisona.

A taxa de infecção por S. mansoni apresentada por moluscos tratados com hidrocortisona (Grupos IV e IVa) foi superior àquela apresentada por $B$. glabrata não tratada (Grupo III e IIIa). Durante os 35 anos que temos mantido em nosso laboratório a linhagem $\mathrm{BH}$ de $S$. 
mansoni, periodicamente reforçada com amostras cedidas pelo Professor Wladimir Lobato Paraense, temos obtido taxas de infecção de B. glabrata, costumeiramente mais elevadas. Contudo observamos que atravessamos períodos em que as taxas de infecção caem aos níveis encontrados no presente trabalho. Até hoje não conseguimos uma explicação para este fenômeno. As primeiras 72 horas de infecção são fundamentais para a determinação da viabilidade das larvas de $S$. mansoni Moluscos tratados com hidrocortisona (Grupo IV) iniciaram a eliminação de cercárias mais precocemente do que moluscos não submetidos ao tratamento (Grupo III) e continuaram a eliminação por um período de tempo maior (até a 19a semana).

Foi altamente significativo o aumento do número de cercárias liberadas pelos moluscos que receberam tratamento com glicocorticóide. Como pode ser observado na Tabela 4, a quantidade máxima de cercárias liberadas semanalmente por moluscos não tratados (Grupo III) não chegou a um quinto do número máximo semanal apresentado pelo grupo de moluscos tratados (Grupo IV). Na mesma tabela, podemos observar ainda que, tais resultados não se devem unicamente ao aumento do número de moluscos susceptíveis, mas também, à presença de alguns moluscos que liberaram número muito elevado de cercárias. É interessante ressaltar que o molusco 3BgIV, aquele que na maioria das vezes apresentou a maior liberação de cercárias por semana no Grupo IV, foi também o que conseguiu manter-se vivo, liberando cercárias, por maior tempo (12 semanas), como pode ser observado na Tabela 4. Tal dado, confirma o favorecimento à sobrevivência dos moluscos submetidos à infecção e tratados com hidrocortisona.

\section{REFERÊNCIAS BIBLIOGRÁFICAS}

1. Barbosa FS, Coelho MV, Dobbin JE. Qualidades de vetor dos hospedeiros de S. mansoni no Nordeste do Brasil. II. Duração da infestação e eliminação de cercárias em A. glabratus. Publicações Avulsas do Instituto Aggeu Magalhães III:79-92, 1954.

2. Guaraldo AMA, Magalhães LA, Rangel HA, Pareja G. Evolução dos esporocistos de Schistosoma mansoni Sambon, 1907 em Biomphalaria glabrata (Say, 1818) e Biomphalaria tenagophila (D’Orbigny, 1835). Revista de Saúde Pública 15:436-448, 1981.

3. Haynes RC. Studies of an in vitro effect of glucocorticoids on gluconeogenesis. Endocrinology 71:399-406, 1962.

4. Landau BR, Mahler R, Ashmore J, Elwyn D, Hasting AB, Zottus $\mathrm{S}$. Cortisone and the regulation of hepatic gluconeogenesis. Endocrinology 70:47-53, 1962.

5. Pan CT. Studies on the host-parasite relationship between Schistosoma mansoni and the snail Australorbis glabratus.
American Journal of Tropical Medicine and Hygiene 14:931-976, 1965.

6. Paraense WL, Corrêa LR. Sobre a ocorrência de duas raças biológicas de Schistosoma mansoni no Brasil. Ciência e Cultura 15:245-246, 1963.

7. Paraense WL, Corrêa LR. A potential vector of Schistosoma mansoni in Uruguay. Memórias do Instituto Oswaldo Cruz 84:281-288, 1989.

8. Pellegrino J, Macedo DG. A simplified method for the concentration of cercariae. Journal of Parasitology 41:329-330, 1955.

9. Stites DP, Terr AI, Parlow TG (eds) Medical Immunology, $9^{\text {th }}$ edition, Appleton \& Lange, Stamford, Connecticut, USA, 1997.

10. Velázquez BL. Terapéutica com sus fundamentos de farmacología experimental. Volume II $11^{\text {a }}$ edición. Editorial Científico-Médica, Barcelona, 1970. 